\title{
Integrated Agent-Based Modeling with GIS for Large Scale Emergency Simulation
}

\author{
Dan Guo, Bo Ren, and Cheng Wang \\ College of Hydropower and Information Engineering, \\ Huazhong University of Science and Technology, \\ 430074 Wuhan, China \\ guodandda126.com
}

L. Kang et al. (Eds.): ISICA 2008, LNCS 5370, pp. 618-625, 2008.

(c) Springer-Verlag Berlin Heidelberg 2008

\section{DOI 10.1007/978-3-540-92137-0_94}

In the original version, the pages $618,619,622,623 \& 625$ don't have the below additional information.

1. On Page 618 of this paper, the sentence "the use of $A B M$ for experimenting and exploring geographical phenomena, specifically linking it to GIS is still in its infancy" was quoted from the paper 110 "Principles and Concepts of Agent-Based Modelling for Developing Geospatial Simulations" by C. J. Castle, and A. T. Crooks, Centre for Advanced Spatial Analysis, Working Paper 110, University College London, 2006.

2. On Page 619, the sentence "ABM is a computational methodology that allows the analysts to create, analyze, and experiment with artificial worlds populated by agents that interact in nontrivial ways" was quoted from the paper "Endogenizing geopolitical boundaries with agent-based modelling" by Lars-Erik Cederman, Proceedings of the National Academy of Sciences of the United States of America, Vol. 99, No. 10, 7294-7303, 2002.

3. Page 622, Fig. 3 was taken from the paper "Constructing and Implementing an Agent-Based Model of Residential Segregation through Vector GIS" by A. T. Crooks, Centre for Advanced Spatial Analysis, Working Paper 133, University College London, 2008.

4. On Page 623, “The open circles are labeled A, V, D, and P, representing program modules which called to animate the walks (A), visualize the geometry and surfaces (V), display numeric data (D), and plot such data (P)." was quoted from the paper "Local Movement: Agent-Based Models of Pedestrian Flow" by Michael Batty, Bin Jiang, and Mark Thurstain Goodwin, Centre for Advanced Spatial Analysis, Working Paper 4, University College London, 1998. 
5. On Page 625, the sentences "Currently, ABM used for simulation analysis has only been applied to abstract systems, which was designed to test how space affects the simulation outcome. Further investigation will apply the models to real world situations and to study issues in detail while testing different scenarios." were quoted from the paper "Exploring Cities using Agent Based Models and GIS" by A. T. Crooks, Proceedings of the Agent 2006 Conference on Social Agents: Results and Prospects, 2006.

6. On Page 625, the sentence "key criteria pertaining to a selection of simulation/modeling systems, is need to be provided to facilitate the identification of a suitable system for the development of a geospatial agent-based model" was quoted from the paper "Principles and Concepts of Agent-Based Modelling for Developing Geospatial Simulations" by C. J. Castle, and A. T. Crooks, Centre for Advanced Spatial Analysis, Working Paper 110, University College London, 2006. 